THURSDAY MARCH 3 I, I870

\section{THE SIZE OF ATOMS}

$T H E$ idea of an atom has been so constantly associated with incredible assumptions of infinite strength, absolute rigidity, mystical actions at a distance, and indivisibility, that chemists and many other reasonable naturalists of modern times, losing all patience with it, have dismissed it to the realms of metaphysics, and made it smaller than "anything we can conceive." But if atoms are inconceivably small, why are not all chemical actions infinitely swift? Chemistry is powerless to deal with this question, and many others of paramount importance, if barred, by the hardness of its fundamental assumptions, from contemplating the atom as a real portion of matter occupying a finite space, and forming a not immeasurably small constituent of any palpable body.

More thän thirty years ago naturalists were scared by a wild proposition of Cauchy's, that the familiar prismatic colours proved the "sphere of sensible molecular action" in transparent liquids and solids to be comparable with the wave-length of light. The thirty years which have intervened have only confirmed that proposition. They have produced a large number of capable judges; and it is only incapacity to judge in dynamical questions that can admit a doubt of the substantial correctness of Cauchy's conclusion. But the "sphere of molecular action" conveys no very clear idea to the non-mathematical mind. The idea which it conveys to the mathematical mind is, in my opinion, irredeemably false. For I have no faith whatever in attractions and repulsions acting at a distance between centres of force according to various laws. What Cauchy's mathematics really proves is this: that in palpably homogeneous bodies such as glass or water, contiguous portions are not similar when their dimensions are moderately small fractions of the wave-length. Thus in water, contiguotis cubes, each of one one-thousandth of a centimetre breadth, are sensibly similar. But contiguous cubes of one ten-millionth of a centimetre must be very sensibly different. So in a solid mass of brickwork, two adjacent lengths of 20,000 centimetres each, may contain, one of them nine hundred and ninety-nine bricks and two half bricks, and the other one thousand bricks: thus two contiguous cubes of 20,000 centimetres breadth may be considered as sensibly similar. But two adjacent lengths of forty centimetres each might contain, one of them one brick and two half bricks, and the other two whole bricks; and contiguous cubes of forty centimetres would be very sensibly dissimilar. In short, optical dynamics leaves no alternative but to admit that the diameter of a molecule, or the distance from the centre of a molecule to the centre of a contiguous molecule in glass, water, or any other of our transparent liquids and solids, exceeds a ten-thousandth of the wavelength, or a two-hundred-millionth of a centimetre.

By experiments on the contact electricity of metals made eight or ten years ago, and described in a letter to Dr. Joule, which was published in the Proceedings of the Literary and Philosophical Society of Manchester, I found that plates of zinc and copper connected with one another by a fine wire attract one another, as would similar pieces of one metal connected with the two plates of a galvanic element, having about three-quarters of the electro-motive force of a Daniel's element.

Measurements published in the Proceedings of the Royal Society for 1860 showed that the attraction between parallel plates of one metal held at a distance apart small in comparison with their diameters, and kept connected with such a galvanic element, would experience an attraction amounting to two ten-thousand-millionths of a gramme weight per area of the opposed surfaces equal to the square of the distance between them. Let a plate of zinc and a plate of copper, each a centimetre square and a hundred-thousandth of a centimetre thick, be placed with a corner of each touching a metal globe of a hundredthousandth of a centimetre diameter. Let the plates, kept thus in metallic communication with one another be at first wide apart, except at the corners touching the little globe, and let them then be gradually turned round till they are parallel and at a distance of a hundred-thousandth of a centimetre asunder. In this position they will attract one another with a force equal in all to two grammes weight. By abstract dynamics and the theory of energy, it is readily proved that the work done by the changing force of attraction during the motion by which we have supposed this position to be reached, is equal to that of a constant force of two grammes weight acting through a space of a hundred-thousandth of a centimetre; that is to say, to two hundred-thousandths of a centimetregramme. Now let a second plate of zinc be brought by a similar process to the other side of the plate of copper; a second plate of copper to the remote side of this second plate of zinc, and so on till a pile is formed consisting of 50,00 I plates of zinc and 50,000 plates of copper, separated by 100,000 spacès, each plate and each space one hundred-thousandth of a centimetre thick. The whole work done by electric attraction in the formation of this pile is two centimetre-grammes.

The whole mass of metal is eight grammes. Hence the amount of work is a quarter of a centimetre-gramme per gramme of metal. Now 4,030 centimetre-grammes of work, according to Joule's dynamical equivalent of heat is the amount required to warm a gramme of zinc or copper by one degree centigrade. Hence the work done by the electric attraction could warm the substance by only $\frac{1}{10} \frac{1}{120}$ of a degree. But now let the thickness of each piece of metal and of each intervening space be a hundred-millionth of a centimetre instead of a hundredthousandth. The work would be increased a millionfold unless a hundred-millionth of a centimetre approaches the smallness of a molecule. The heat equivalent would therefore be enough to raise the temperature of the material by $62^{\circ}$. This is barely, if at all, admissible, according to our present knowlêdge, or, rather, want of knowledge, regarding the heat of combination of zinc and copper. But suppose the metal plates and intervening spaces to be made yet four times thinner, thiat is to say, the thickness of each to be four-hundred-millionth of a centimetre. The work and its heat equivalent will be increased sixteen-fold. It would therefore be 990 times as much as that required to warm the mass by to cent., which is very much more than can possibly be produced by zinc and copper in entering into molecular combination. Were there in reality anything like so much heat 
of combination as this, a mixture of zinc and copper powders would, if melted in any one spot, run together, generating more than heat enough to melt each throughout; just as a large quantity of gunpowder if ignited in any one spot burns throughout without fresh application of heat. Hence plates of zinc and copper of a threehundred-millionth of a centimetre thick, placed close together alternately, form a near approximation to a chemical combination, if indeed such thin plates could be made without solitting atoms.

The theory of capillary attraction shows that when a bubble-a soap-bubble for instance-is blown larger and larger, work is done by the stretching of a film which resists extension as if it were an elastic membrane with a constant contractile force. This contractile force is to be reckoned as a certain number of units of force per unit of breadth. Observation of the ascent of water in capillary tubes shows that the contractile force of a thin film of water is about sixteen milligrammes weight per millimetre of breadth. Hence the work done in stretching a water film to any degree of thinness, reckoned in millimetremilligrammes, is equal to sixteen times the number of square millimetres by which the area is augmented, provided the film is not made so thin that there is any sensible diminution of its contractile force. In an article "On the Thermal effect of drawing out a Film of Liquid," published in the Proceedings of the Royal Society for April I 858 , I have proved from the second law of thermodynamics that about half as much more energy, in the shape of heat, must be given to the film to prevent it from sinking in temperature while it is being drawn out. Hence the intrinsic energy of a mass of water in the shape of a film kept at constant temperature increases by twenty-four milligramme-millimetres for every square millimetre added to its area.

Suppose then a film to be given with a thickness of a millimetre, and suppose its area to be augmented ten thousand and one fold: the work done per square millimetre of the original film, that is to say per milligramme of the mass, would be 240,000 millimetre-milligrammes. The heat equivalent of this is more than half a degree centigrade of elevation of temperature of the substance. The thickness to which the film is reduced on this supposition is very approximately a ten-thousandth of a millimetre. The commonest observation on the soap-bubble (which in contractile force differs no doubt very little from pure water) shows that there is no sensible diminution of contractile force by reduction of the thickness to the tenthousandth of a millimetre; inasmuch as the thickness which gives the first maximum brightness round the black spot seen where the bubble is thinnest, is only about an eight-thousandth of a millimetre.

The very moderate amount of work shown in the preceding estimates is quite consistent with this deduction. But suppose now the film to be further stretched, until its thickness is reduced to a twenty-millionth of a millimetre. The work spent in doing this is two thousand times more than that which we have just calculated. The heat equivalent is $I, I 30$ times the quantity required to raise the temperature of the liquid by one degree centigrade. This is far more than we can admit as a possible amount of work done in the extension of a liquid film. A smaller amount of work spent on the liquid would convert it into vapour at ordinary atmospheric pressure. The conclusion is unavoidable, that a water-film falls off greatly in its contractile force before it is reduced to a thickness of a twenty-millionth of a millimetre. It is scarcely possible, upon any conceivable molecular theory, that there can be any considerable falling off in the contractile force as long as there are several molecules in the thickness. It is therefore probable that there are not several molecules in a thickness of a twenty-millionth of a millimetre of water.

The kinetic theory of gases suggested a hundred years ago by Daniel Bernouilli has, during the last quarter of a century, been worked out by Herapath; Joule, Clausius, and Maxwell, to so great perfection that we now find in it satisfactory explanations of all nonchemical properties of gases. However difficult it may be even to imagine what kind of thing the molecule is, we may regard it as an established truth of science that a gas consists of moving molecules disturbed from rectilineal paths and constant velocities by collisions or mutual influences, so rare that the mean length of proximately rectilineal portions of the path of each molecule is many times greater than the average distance from the centre of each molecule to the centre of the molecule nearest it at any time. If, for a moment, we suppose the molecules to be hard elastic globes all of one size, influencing one another only through actual contact, we have for each molecule simply a zigzag path composed of rectilineal portions, with abrupt changes of direction. On this supposition Clausius proves, by a simple application of the calculus of probabilities, that the average length of the free path of a particle from collision to collision bears to the diameter of each globe, the ratio of the whole space in which the globes move, to eight times the sum of the volumes of the globes. It follows that the number of the globes in unit volume is equal to the square of this ratio divided by the volume of a sphere whose radius is equal to that average length of free path. But we cannot believe that the individual molecules of gases in general, or even of any one gas, are hard elastic globes. Any two of the moving particles or molecules must act upon one another somehow, so that when they pass very near one another they shall produce considerable deflexion of the path and change in the velocity of each. This mutual action (called force) is different at different distances, and must vary, according to variations of the distance, so as to fulfil some definite law. If the particles were hard elastic globes acting upon one another only by contact, the law of force would be-zero force when the distance from centre to centre exceeds the sum of the radii, and infinite repulsion for any distance less than the sum of the radii. This hypothesis, with its "hard and fast" demarcation between no force and infinite force, seems to require mitigation. Without entering on the theory of vortex atoms at present, I may at least say that soft elastic solids, not necessarily globular, are more promising than infinitely hard elastic globes. And, happily, we are not left merely to our fancy as to what we are to accept for probable in respect to the law of force. If the particles were hard elastic globes, the average time from 
collision to collision would be inversely as the average velocity of the particles. But Maxwell's experiments on the variation of the viscosities of gases with change of temperature prove that the mean time from collision to collision is independent of the velocity, if we give the name collision to those mutual actions only which produce something more than a certain specified degree of deflection of the line of motion. This law could be fulfilled by soft elastic particles (globular or not globular); but, as we have seen, not by hard elastic globes. Such details, however, are beyond the scope of our present argument. What we want now is rough approximations to absolute values, whether of time or space or mass-not delicate differential results. By Joule, Maxwell, and Clausius we know that the average velocity of the molecules of oxygen or nitrogen or common air, at ordinary atmospheric temperature and pressure, is about 50,000 centimetres per second, and the average time from collision to collision a five-thousand-millionth of a second. Hence the average length of path of each molecule beween collisions is about $\frac{1}{100000}$ of a centimetre. Now, having left the idea of hard globes, according to which the dimensions of a molecule and the distinction between collision and no collision are perfectly sharp, something of apparent circumlocution must take the place of these simple terms.

First, it is to be remarked that two molecules in collision will exercise a mutual repulsion in virtue of which the distance between their centres, after being diminished to a minimum, will begin to increase as the molecules leave one another. This minimum distance would be equal to the sum of the radii, if the molecules were infinitely hard elastic spheres; but in reality we must suppose it to be very different in different collisions. Considering only the case of equal molecules, we might, then, define the radius of a molecule as half the average shortest distance reached in a vast number of collisions. The definition I adopt for the present is not precisely this, but is chosen so as to make as simple as possible the statement I have to make of a combination of the results of Clausius and Maxwell. Having defined the radius of a gaseous molecule, I call the double of the radius the diameter; and the volume of a globe of the same radius or diameter I call the volume of the molecule.

The experiments of Cagniard de la Tour, Faraday, Regnault, and Andrews, on the condensation of gases do not allow us to believe that any of the ordinary gases could be made forty thousand times denser than at ordinary atmospheric pressure and temperature, without reducing the whole volume to something less than the sum of the volume of the gaseous molecules, as now defined. Hence, according to the grand theorem of Clausius quoted above, the average length of path from collision to collision cannot be more than five thousand times the diameter of the gaseous molecule; and the number of molecules in unit of volume cannot exceed $25,000,000$ divided by the volume of a globe whose radius is that average length of path. Taking now the preceding estimate, $\frac{1}{100000}$ of a centimetre, for the average length of path from collision to collision, we conclude that the diameter of the gaseous molecule cannot be less than B0000000 of a centimetre; nor the number of molecules in a cubic centimetre of the gas (at ordinary density) greater than $6 \times$ IO $^{2 x}$. (or six thousand million million million).

The densities of known liquids and solids are from five hundred to sixteen thousand times that of atmospheric air at ordinary pressure and temperature; and, therefore, the number of molecules in a cubic centimetre may be from $3 \times 10^{24}$ to $10^{26}$ (that is, from three million million million million to a hundred million million million million). From this (if we assume for a moment a cubic arrangement of molecules), the distance from centre to nearest centre in solids and liquids may be estimated at from 1.5000000 to $\frac{1}{400000000}$ of a centimetre.

The four lines of argument which $I$ have now indicated, lead all to substantially the same estimate of the dimensions of molecular structure. Jointly they establish with what we cannot but regard as a very high degree of probability the conclusion that, in any ordinary liquid, transparent solid, or seemingly opaque solid, the mean distance between the centres of contiguous molecules is less than the hundred-millionth, and greater than the two thousandmillionth of a centimetre.

To form some conception of the degree of coarsegrainedness indicated by this conclusion, imagine a rain drop, or a globe of glass as large as a pea, to be magnified up to the size of the earth, each constituent molecule being magnified in the same proportion. The magnified structure would be coarser grained than a heap of small shot, but probably less coarse grained than a heap of cricket-balls.

W. T.

\section{FRESENIUS'S ANALYSIS}

Qualitative Chemical Analysis. By Dr. C. Remigius Fresenius. Seventh Edition. Edited by Arthur Vacher. 8vo., pp. viii. and 264. (London: Churchill, 1869.)

Quantitative Chemical Analysis. By Dr. C. Remigius Fresenius. Fifth edition. Edited by Arthur Vacher. 8 vo., pp. viii. and 377. (London: Churchill, 1870.)

Anleitung zur qualitativen chemischen Analyse. By Dr. C. Remigius Fresenius. 8vo., pp. xii. and 240 , with 43 woodcuts; price $4 s$. (Brunswick, 1869. London: Williams and Norgate.)

IN no branch of chemistry, perhaps, has more useful progress been made of late years than in analysis. The other departments of the science, technical and organic chemistry, for instance, have been cultivated with assiduity and even ostentation; while the study of analysis, invaluable and necessary as it is, has been comparatively neglected as humble and unadorned. Much of this apathy has no doubt arisen from the mechanical nature of the task of analytical discovery, which commonly requires a greater share of industry than intellectual effort. The gradual introduction of refined physical methods has, however, commenced, and will no doubt complete an entire change in the aspect of this subject.

Chemists will not need to be reminded of the obligations they owe to Fresenius, whose analytical manuals are deservedly known, and in common use in almost every laboratory. The enormous amount of special results they contain is hardly conceivable to an outsider, who will not readily appreciate the respect paid to them by grateful 